\title{
QUELQUES RÉSULTATS \\ SUR LES APTITUDES ZOOTEGHNIQUES DES BREBIS DE RACE LACAUNE
}

PAR

\section{H. ANGEL, P. CHARLET, J. POLY}

Laboratoire de Recherches de Zootechnie, Institut National Agronomique, Paris

Au moment où les Pouvoirs publics et les Associations d'élevage se préoccupent du contrôle des aptitudes zootechniques des ovins, nous avons jugé intéressant de publier les résultats d'une enquête - malheureusement incomplète - réalisée, il y a un certain nombre d'années, concernant les productions lainière et laitière des brebis de race Lacaune.

On avait choisi Montlaur, dans le Camarès, comme centre d'études, car la région qui l'entoure vit exclusivement de la production du lait de brebis; de plus, le contrôle laitier y est assez développé. Les animaux appartiennent en général à de petits troupeaux, mais les conditions d'élevage sont relativement uniformes dans la région. Les brebis, nourries l'hiver en bergerie, reçoivent une ration à base de luzerne, de céréales, exceptionnellement complétée par des tourteaux. Pendant la belle saison, les animaux vivent sur les parcours du Camarès. La lactation commence en janvier.

En I946, on avait prélevé des échantillons de laine à l'épaule et à la cuisse de 58 brebis laitières. Ces échantillons furent étudiés par le Laboratoire d'analyses et de recherches industrielles de Roubaix.

Les résultats obtenus en ce qui concerne la finesse, la longueur, la résistance des brins, sont consignés dans le tableau I.

\section{TABLEAU I}

Caractéristiques lainières de 58 brebis de race Lacaune

\begin{tabular}{|c|c|c|c|c|c|c|}
\hline \multirow[b]{2}{*}{ Caractéristiques } & \multicolumn{2}{|c|}{ Epaule } & \multicolumn{2}{|c|}{ Cuisse } & \multicolumn{2}{|c|}{ Mloyenne épaule-cuisse } \\
\hline & Moyenne & $\mid \begin{array}{c}\text { Coeffi- } \\
\text { cient de } \\
\text { variation }\end{array}$ & Moyenne & $\mid \begin{array}{c}\text { Coeffi- } \\
\text { cient de } \\
\text { variation }\end{array}$ & Moyenne & $\begin{array}{l}\text { Coeffi- } \\
\text { cient de } \\
\text { variation }\end{array}$ \\
\hline \multirow{3}{*}{$\begin{array}{l}\text { Finesse en Microns } \\
\text { Longueur étirée en } \\
\mathrm{cm} . . . \ldots . \ldots . . . \\
\text { Résistance à la rup- } \\
\text { ture en gr ...... }\end{array}$} & $26,92 \pm 0,4^{2}$ & 0,120 & $28, \mathrm{I} 7 \pm 0,25$ & 0,067 & $27,54 \pm 0,39$ & 0,108 \\
\hline & $5,77 \pm 0,16$ & $0,2 \mathrm{I} 3$ & $5,71 \pm 0,19$ & 0,257 & $5,71 \pm 0,16$ & 0,218 \\
\hline & ${ }^{1} 1,33 \pm 0,3^{6}$ & 0,239 & $12,23 \pm 0,43$ & 0,270 & I $1,78 \pm 0,3^{8}$ & 0,245 \\
\hline
\end{tabular}


L,es brebis laitières de race Lacaune ont donc une toison de finesse moyenne comparable à celle des moutons de race Ile-de-France. (Ainsi, la finesse moyenne PX/XI (Prime croisé-croisé I) indiquée dans les " standards laine" (I) des principales races françaises semble un peu trop élevée pour nos données.) En outre, des valeurs extrêmes individuelles de 22,4 et 35,3 microns traduisent, à notre avis, une assez grande hétérogénéité dans la race pour ce caractère.

La mèche est courte ; sur ce point, nous sommes en désaccord avec les chiffres avancés par PORTAL et QUITTET (2) qui indiquent une longueur moyenne des brins, comprise entre Io $\mathrm{cm}$ et $\mathrm{I}_{5} \mathrm{~cm}$; de plus, la longueur moyenne varie considérablement d'une brebis à l'autre, car nous avons relevé des valeurs extrêmes de $3,4 \mathrm{~cm}$ et II $\mathrm{cm}$.

A l'aide des mêmes données, nous avons calculé la corrélation phénotypique existant entre la finesse moyenne et la longueur de mèche moyenne d'une brebis:

$r=+0,397$, résultat hautement significatif. On retrouve ce fait bien connu que les mèches les plus fines ont tendance à être les plus courtse.

En 1947 et en I948, on a étudié systématiquement les productions zootechniques des brebis de six troupeaux de la région de Montlaur en relation avec leurs conditions de milieu: habitat, alimentation ( ${ }^{1}$ ).

A la tonte du printemps I948, on a dressé pour 73 brebis appartenant à ces six troupeaux, une fiche mentionnant:

le nom de l'élevage ;

le numéro d'oreille et le numéro de Flock-Book ;

la production laitière contrôlée ;

la durée de lactation contrôlée ;

le poids de la toison;

le périmètre thoracique.

Un schéma permettait également d'estimer 1' "indice de couverture " de l'animal, c'est-à-dire l'étendue de sa toison.

Le tableau II rassemble les moyennes de la durée de lactation, de

TABLEaU II

\begin{tabular}{|c|c|c|c|c|c|}
\hline Elevages & $\begin{array}{l}\text { Nombre } \\
\text { de brebis }\end{array}$ & $\begin{array}{l}\text { Durée } \\
\text { de lactation }\end{array}$ & $\begin{array}{c}\text { Production laitière } \\
(\mathrm{kg})\end{array}$ & $\begin{array}{l}\text { Poids de toison } \\
\text { (kg) }\end{array}$ & $\begin{array}{l}\text { Périmètre } \\
\text { thoracique } \\
\text { (m) }\end{array}$ \\
\hline & 18 & I 59,9 & IIo,34 上 4,39 & $\mathrm{I}, 487 \pm 0,106$ & $0,937 \pm 0,0086$ \\
\hline & 12 & 180,0 & $150,01 \pm 5,59$ & $\mathrm{I}, 23 \mathrm{I} \pm 0, \mathrm{I} 67$ & $0,934=0,0098$ \\
\hline C. & 12 & 157,9 & $127,47=6,8 \mathrm{I}$ & $\mathrm{I}, 92 \mathrm{I} \pm 0,186$ & $0,94^{2} \pm 0,0138$ \\
\hline & 7 & 170,0 & $13^{8,72}=8,95$ & $\mathrm{I}, 300 \pm 0,13^{8}$ & $0,943 \pm 0,0182$ \\
\hline E. & I 2 & 180,1 & I $17,42 \pm 6,59$ & $\mathrm{I}, \mathrm{I} 83 \pm 0,142$ & $0,968 \pm 0,0.160$ \\
\hline & 12 & 160,2 & $127,83 \pm 9,99$ & $\mathrm{I}, 654 \pm 0,178$ & $0,972 \pm 0,0115$ \\
\hline Total & 73 & 167,2 & $126,44 \pm 3,16$ & $I, 476 \pm 0,068$ & $0,949 \pm 0,0052$ \\
\hline
\end{tabular}

(1) Ce travail d'enquête fut conduit à l'époque par Mile PAILlard, ingénieur agronome. 
la production laitière, du poids de toison et du périmètre thoracique par troupeau, et pour l'ensemble des 73 brebis.

Les analyses de variance qui figurent au tableau III ont permis d'étudier les différences existant entre les six troupeaux pour les trois caractères considérés.

TABLEAU III

\begin{tabular}{|c|c|c|c|c|c|c|c|}
\hline \multirow{2}{*}{ Sources de variance } & \multirow{2}{*}{$\begin{array}{l}\text { Degrés } \\
\text { de liberté }\end{array}$} & \multicolumn{2}{|c|}{ Production laitière } & \multicolumn{2}{|c|}{ Poids de toison } & \multicolumn{2}{|c|}{$\begin{array}{l}\text { Périmètre } \\
\text { thoracique }\end{array}$} \\
\hline & & $\begin{array}{c}\text { Somme } \\
\text { des carrés }\end{array}$ & Variance & \begin{tabular}{|} 
Somme \\
des carrés
\end{tabular} & Variance & $\begin{array}{c}\text { Somme } \\
\text { des carrés }\end{array}$ & Variance \\
\hline \multirow[t]{2}{*}{$\begin{array}{l}\text { Variance totale ......... } \\
\text { Entre troupeaux ........ } \\
\text { A l'intérieur des troupeaux }\end{array}$} & \multirow[t]{2}{*}{$\begin{array}{r}72 \\
5 \\
67\end{array}$} & $\left|\begin{array}{l}52.599,76 \\
13.400,77 \\
39.198,99\end{array}\right|$ & \begin{tabular}{|l}
$2.680, \mathrm{I}$ \\
$5^{8} 5,05$
\end{tabular} & $\begin{array}{r}24,1426 \\
4,7234 \\
19,4191\end{array}$ & $\begin{array}{l}0,9447 \\
0,2898\end{array}$ & $\begin{array}{l}0, \mathrm{I} 433 \\
0,0170 \\
0,1263\end{array}$ & $\begin{array}{l}0,0034 \\
0,001885\end{array}$ \\
\hline & & \multicolumn{2}{|c|}{$\mathrm{F}=\frac{2.680, \mathrm{I}}{5^{85}, 05}=\underset{(\mathrm{HS})}{4,58}$} & \multicolumn{2}{|c|}{$\mathrm{F}=\frac{0,9447}{0,2898}=3,26$} & \multicolumn{2}{|c|}{$\mathrm{F}=\frac{0,0034}{0,001885}=\mathrm{T}, \mathrm{S}$} \\
\hline
\end{tabular}

NS : non significatif $-\mathrm{S}$ : significatif (seuil $5 \%$ ) - HS : hautement significatif (seuil I \%).

Les troupeaux ne diffèrent pas les uns des autres en ce qui concerne le périmètre thoracique, c'est-à-dire que les animaux ont un format qui ne varie pas plus d'un élevage à l'autre que parmi les brebis d'un même troupeau.

On a trouvé en effet qu'il existait une relation très étroite, chez les ovins comme chez les bovins, entre le périmètre thoracique et le poids vif des animaux. Les études de RaGab, Asker et Youssef (3) sur les races ovines égyptiennes Ossimi et Rahmani sont particulièrement démonstratives à cet égard.

Au contraire, les différences significatives et hautement significatives entre élevages en ce qui concerne la production laitière et le poids de toison sont assez surprenantes. Elles doivent être la conséquence, d'une part des conditions d'entretien des troupeaux, affourragement notamment et, d'autre part, de la valeur génétique des béliers utilisés pour la lutte, valeur que l'on ne peut chiffrer dans les conditions actuelles de 1'élevage.

La corrélation phénotypique entre la production laitière et le poids de toison a été calculée sur l'ensemble des données:

$r=0, \mathrm{I} 66$, résultat non significatif.

Ce résultat diffère nettement de celui $(r=+0,50)$ que AbIBon et col. (4) avaient calculé à partir des données de 42 brebis laitières. A la réflexion, des résultats aussi différents n'ont pas lieu de surprendre. En effet, dans ces deux études, le coeffcient de corrélation mesure l'intensité de liaison entre le phénotype production laitière et le phénotype poids de la toison, et non les relations génétiques entre ces deux types de per- 
formances. MASON insistait à juste titre sur ce point aux Journées du Mouton (5).

Dans une étude sur la production des brebis laitières italiennes de la race de Langhe, Mason et DAssat (6) ont calculé la corrélation entre la production laitière et le poids de toison à l'intérieur des troupeaux, de plusieurs façons. Les résultats figurent ci-dessous :

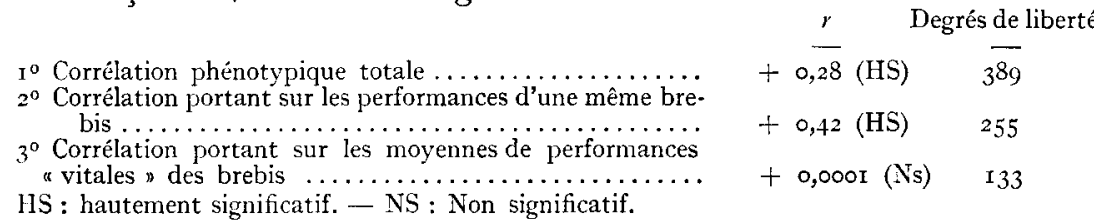

(L,es moyennes de performances « vitales» sont définies d'une part par la moyenne des productions laitières correspondant aux différentes lactations et, d'autre part, par la moyenne du poids de toison correspondant aux différentes tontes enregistrées au cours de la vie de chaque brebis.)

Selon les deux auteurs, la corrélation phénotypique totale inclut, non seulement les relations génétiques existant entre les deux types de performances, mais aussi celles qui sont dues aux effets permanents et temporaires du milieu. La deuxième corrélation, au contraire, est due uniquement aux effets temporaires du milieu; elle a été calculée à partir des performances des mêmes brebis sur plusieurs années. Mason et DASSAT concluent que " la corrélation nulle entre les moyennes " vitales" des deux types de performances est la résultante d'une corrélation génétique et d'une corrélation due aux effets permanents du milieu. Si cette dernière est positive (ce qui semblerait vrai, par analogie avec la corrélation due aux effets temporaires du milieu, c'est-à-dire si les conditions favorables in utero et dans le jeune âge améliorent à la fois la production laitière et le poids de toison à l'âge adulte) la corrélation génétique doit être alors négative" .

La comparaison des différents coefficients de corrélation seule permet donc de se faire une idée du sens et de l'intensité des divers types de liaison existant entre deux caractères. Et des corrélations positives élevées, comme celle que MM. Aвгвon et col. (4) ont calculée, peuvent s'expliquer par des effets temporaires du milieu ayant influencé favorablement à la fois les deux caractères étudiés. Il est fort possible, d'autre part, que la dispersion des valeurs en nombre insuffisant sur une assez large échelle fasse apparaître des corrélations élevées fictives.

On peut invoquer des raisons physiologiques pour expliquer la corrélation laine-lait.

HAmmond (7) a émis 1'idée d'une compétition pour les éléments nutritifs véhiculés par le sang entre les principales productions zootechniques. Une récente étude effectuée par LEROY et col. (8) sur la pousse de la laine chez des moutons Ile-de-France a montré un affinement des 
brins et une réduction de la quantité journalière de laine poussée pendant la période de lactation, ce qui semble indiquer que la production laitière est prioritaire sur la production de laine. Ces résultats sont, d'ailleurs, en accord avec ceux qui se dégagent d'un grand nombre de travaux ou d'observations sur cette même question.

Nous avons essayé d'étudier les relations existant entre 1' "indice de couverture " d'une part et la production de lait et le poids de toison d'autre part. Il est bien connu que l'indice de couverture ou fraction de la surface corporelle qui est recouverte par de la laine varie considérablement d'une race à l'autre. CHARLE'T (9) donnait récemment quelques valeurs moyennes pour différentes races françaises et notamment un indice de couverture de $60 \mathrm{p}$. Ioo pour le Lacaune. Ce chiffre est, pour nos données, trop élevé. A l'intérieur de cette race, il existe, de plus, une très grande variabilité de ce caractère, ce qui n'est pas le cas, par exemple, dans le Mérinos de Rambouillet ou dans l'Ile-de-France.

Ainsi, nous avons réparti les 73 brebis étudiées en cinq classes ; la classe I correspond aux animaux les plus découverts et la classe $\mathrm{V}$ aux animaux les plus couverts en laine. Le tableau IV indique les moyennes des productions laitières, des poids de toison et des périmètres thoraciques pour ces cinq classes.

TABleaU IV

\begin{tabular}{|c|c|c|c|c|}
\hline Classes & $\begin{array}{l}\text { Nombre } \\
\text { de brebis }\end{array}$ & $\underset{(\mathrm{kg})}{\text { Production de lait }}$ & $\begin{array}{c}\text { Poids de toison } \\
(\mathrm{kg})\end{array}$ & $\begin{array}{l}\text { Périmètre thorac. } \\
(\mathrm{m})\end{array}$ \\
\hline I . . & 10 & $\mathrm{I} 28,47 \pm 10,5 \mathrm{I}$ & $0,9 \mathbf{I} 5 \pm 0,08 \mathbf{I}$ & $0,969 \pm 0,015$ \\
\hline$\ldots \ldots \ldots$ & 17 & $131,20 \pm 7,90$ & $1,322 \pm 0,126$ & $0,935 \pm 0,009$ \\
\hline$\ldots \ldots \ldots \ldots \ldots$ & 26 & $125,09 \pm 4,99$ & $1,377 \pm 0,073$ & $0,948 \pm 0,009$ \\
\hline$\ldots \ldots \ldots \ldots \ldots$ & 13 & $122,23 \pm 6,07$ & $1,792 \pm 0, \operatorname{IO9}$ & $0,957 \pm 0,013$ \\
\hline$V \ldots \ldots \ldots \ldots$ & 7 & $\mathrm{I} 24,83 \pm 9,08$ & $2,429 \pm 0,23^{6}$ & $0,944 \pm 0,014$ \\
\hline Total. . . . . . . . . & 73 & $126,44 \pm 3,16$ & $1,47^{6} \pm 0,068$ & $0,949 \pm 0,005$ \\
\hline
\end{tabular}

Les analyses de variance présentées dans le tableau $\mathrm{V}$ permettent d'étudier les différences existant entre les diverses classes en ce qui concerne la production laitière, le poids de toison et le périmètre thoracique.

TABLEAU V

\begin{tabular}{|c|c|c|c|c|c|c|c|}
\hline \multirow{2}{*}{ Sources de variance } & \multirow{2}{*}{$\begin{array}{l}\text { Degrés } \\
\text { de liberté }\end{array}$} & \multicolumn{2}{|c|}{ Production laitière } & \multicolumn{2}{|c|}{ Poids de toison } & \multicolumn{2}{|c|}{$\begin{array}{l}\text { Périmètre } \\
\text { thoracique }\end{array}$} \\
\hline & & $\begin{array}{c}\text { Somme } \\
\text { des carrés }\end{array}$ & Variance & $\begin{array}{c}\text { Somme } \\
\text { des carrés }\end{array}$ & Variance & $\begin{array}{c}\text { Somme } \\
\text { des carrés }\end{array}$ & Variance \\
\hline \multirow[t]{2}{*}{$\begin{array}{l}\text { Variance totale } \ldots \ldots \ldots \\
\text { Entre classes } \ldots \ldots \ldots \\
\text { Entre individus d'une } \\
\text { même classe .......... }\end{array}$} & $\begin{array}{r}72 \\
4 \\
68\end{array}$ & $\begin{array}{r}52.599,76 \\
721,85 \\
51.877,91\end{array}$ & $\begin{array}{l}180,46 \\
762,9\end{array}$ & $\begin{array}{l}24, \mathrm{I} 426 \\
\mathrm{II}, 4573 \\
\text { I } 2,6853\end{array}$ & $\begin{array}{l}2,864 \\
0,1865\end{array}$ & $\begin{array}{l}0, \mathrm{I} 433 \\
0,0084 \\
0, \mathrm{I} 349\end{array}$ & $\begin{array}{l}0,0021 \\
0,00198\end{array}$ \\
\hline & & $\mathrm{F}<\mathrm{I}$ & (NS) & $F=I_{5}, 3$ & $3^{6} \quad(\mathrm{HS})$ & $\mathrm{F}=\mathrm{I}, \mathrm{I}$ & $6 \quad(\mathrm{NS})$ \\
\hline
\end{tabular}

NS : non significatif - HS : hautement significatif (seuil r \%). 
Les diverses classes sont comparables quant au format des brebis: Les groupes considérés ne diffèrent pas les uns des autres au point de vue production laitière, ce qui vient contredire l'opinion couramment admise et rapportée récemment encore par PUEch (ro) aux Journées du Mouton, selon laquelle les animaux les moins couverts de laine sont les plus laitiers. Par contre, il y a une différence hautement significative entre les classes en ce qui concerne le poids de la toison, les brebis ayant une toison d'autant plus lourde que leur indice de couverture est plus élevé.

L'analyse de variance du tableau $\mathrm{V}$ a permis d'évaluer la part de la variance du poids de toison que l'on peut attribuer à la variation de l'indice de couverture; nous avons trouvé que 5I,4 p. Ioo de la variance totale du poids de toison est due à l'indice de couverture.

On remarquera la part importante prise par l'étendue de la toison dans le poids total de laine récolté à la tonte sur ces brebis.

Il n'en est pas ainsi dans la plupart des autres races ovines françaises chez lesquelles l'indice de couverture ne variant que peu d'un animal à l'autre, le poids de la toison dépend essentiellement du tassé, c'est-à-dire de la densité d'implantation des fibres de laine et des caractéristiques de ces brins, principalement de leur longueur moyenne.

\section{RÉSUMÉ}

Une étude sur les aptitudes zootechniques des brebis de race Iracaune a été réalisée il y a quelques années.

Les moyennes de finesse et de longueur de mèches prélevées à l'épaule et à la cuisse de 58 brebis étaient de 27,54 microns et de $5,74 \mathrm{~cm}$ respectivement. Le coefficient de corrélation liant ces deux caractères était de $+0,397$. La résistance moyenne à la rupture des brins de laine était de $I I, 78 \mathrm{~g}$.

D'après une étude portant sur 73 brebis Iacaune appartenant à six élevages de la région de Montlaur, Aveyron, la production laitière moyenne contrôlée par animal était de $126,44 \mathrm{~kg}$ pour une durée de lactation de $I 67,2$ jours. Le poids moyen de toison était de $\mathrm{I}, 476 \mathrm{~kg}$.

Des différences significatives existaient entre troupeaux en ce qui concerne la production laitière et le poids de toison. Les élevages étaient semblables quant au format de leurs brebis, comme le montre l'analyse de variance du périmètre thoracique. La variation des performances de troupeau à troupeau doit s'expliquer par des différences dans les conditions d'alimentation et dans la valeur des béliers utilisés pour la lutte.

Le coefficient de corrélation phénotypique entre production laitière et poids de toison était négatif, mais non significativement différent de zéro. 
Les animaux, différant notablement les uns des autres en ce qui concerne 1'étendue de la toison, furent divisés en cinq classes selon 1' " indice de couverture ". La variation de 1'étendue de la toison constituait $5 \mathrm{I}, 4 \mathrm{p}$. Ioo de la variance totale du poids de toison.

Une étude plus poussée devrait être poursuivie pendant plusieurs années sur les mêmes animaux; d'autres caractéristiques comme le taux butyreux du lait, la finesse des brins de laine, la longueur de mèche et le tassé des toisons devraient également être enregistrées ; cela permettrait de définir l'influence de certains facteurs du milieu et d'obtenir une meilleure connaissance des performances lainière et laitière des brebis de race Lacaune.

\section{Remerciements :}

Nous tenons à remercier la Fédération Nationale Ovine et le Comité National Interprofessionnel de la Laine dont l'aide matérielle a permis la réalisation de ces travaux.

\section{RÉFÉRENCES BIBLIOGRAPHIQUES}

(I) COMITÉ NATIONAL INTERPRofESSIONNEL DE LA LAINE. - Les “ standardslaine " des principales races françaises ; p. 8.

(2) Portal (M.), QuitTet (E.). - Les races ovines françaises; r95o; p. 54.

(3) Ragab (M. T.), Asker (A. A.), Youssef (A. A.). - Comparative study of body weight and measurements of Ossimi and Rahamani sheep. Faculty of A griculture, Cairo University ; I953; Bulletin 27 ; p. 3-4I.

(4) Abibon, Dobignies, Ortavant, ValdebouzE. - Sur la corrélation entre la production du lait et celle de la laine des brebis de Lacaune. Comptes rendus de l'Académie d'A griculture; $1948 ; \mathrm{n}^{\circ} 2$; p. I03-ro8.

(5) Mason (I.). - Programme de sélection en matière d'élevage ovin. Bulletin Technique d'Information; $1953 ; \mathrm{n}^{\circ} 76 ; \mathrm{p} .7 \mathrm{I}$.

(6) Mason (I.), Dassat (P.). - Milk, meat and wool production in the Langhe sheep of Italy. Zeitschrift für Tierziichtung und Zïchtungsbiologie; I954; Band 62, Heft 3, p. I97-234.

(7) Hammond (J.). - Physiological limits to intensive production in animals. British A gricultural Bulletin; I952; vol. 4 ; $\mathrm{n}^{\circ}$ I6 ; p. 223.

(8) I.EROY (M.) et collaborateurs. - Etude de la pousse de la laine dans les conditions pratiques de l'élevage. A paraître dans les Annales de Zootechnie, de 1'I.N.R.A.

(9) Charlete (P.). - Méthode directe d'appréciation du tassé des toisons. Annales de Zootechnie de 1'I.N.R.A. ; I952; $\mathrm{n}^{\circ} \mathrm{I} ;$ p. 2.

(ro) PUECH (G.). - Orientation et amélioration de la production du lait de brebis. Bulletin Technique d'Information; $1953 ; \mathrm{n}^{\mathrm{O}} 76 ; \mathrm{p} .4 \mathrm{I}$. 